\title{
Postoperative cognitive dysfunction - current preventive strategies
}

This article was published in the following Dove Press journal:

Clinical Interventions in Aging

\author{
Nalini Kotekar' \\ Anshul Shenkar ${ }^{2}$ \\ Ravishankar Nagaraj ${ }^{3}$ \\ 'Department of Anaesthesiology, \\ JSS Academy of Higher Education and \\ Research, Mysore, Karnataka, India; \\ ${ }^{2}$ Department of Anaesthesiology, \\ AJ Medical College and Research \\ Centre, Mangalore, Karnataka, India; \\ ${ }^{3}$ Department of Surgery, JSS Academy \\ of Higher Education and Research, \\ Mysore, Karnataka, India
}

\begin{abstract}
Improving trends in global health care have resulted in a steady increase in the geriatric population. However, as the population ages, surgery is being performed more frequently in progressively older patients and those with higher prevalence of comorbidities. A significant percentage of elderly patients experience transient postoperative delirium following surgery or long-term postoperative cognitive dysfunction (POCD). Increasing age, educational level, pre-existing mental health, and comorbidities are contributory factors. Comprehensive geriatric assessment provides an objective evaluation on overall medical, social, mental, and functional well-being with scope for preoperative optimization. Preventive strategies for POCD target the surgical and patient-related factors as well as the utilization of the concept of stress-free anesthesia and surgery, that is, Enhanced Recovery After Surgery. This includes care bundles and protocols for the perioperative period which improves outcomes in the elderly. Research on biomarkers of neural injury in POCD is gaining momentum. Pharmacologic agents such as acetylcholine esterase inhibitors promise to have a vital role in the management of POCD but exhibit undesired side effects. Interventions to reduce oxidative stress and neuroinflammation could prove beneficial. Preventive strategies, early recognition, and management of perioperative risk factors seems to be, by far, the best modality to deal with POCD till further progress in therapeutic interventions evolve.
\end{abstract}

Keywords: cognitive dysfunction, geriatric anesthesia, neuronal injury, neuroinflammation, enhanced recovery, prehabilitation

\section{Background}

Demography shows that the number of "old olds" (75-84 years) and oldest (85+) population is on a steady rise due to improvement in general living standards, health care, nutrition, and education. ${ }^{1}$ Globally $50 \%$ of all the elderly individuals are estimated to undergo at least one surgical procedure, and the changes that accompany aging, in turn, influence the individual's ability to withstand the stress of injury, surgery, and anesthesia. Postoperative cognitive dysfunction (POCD) is a condition that has been poorly defined yet recognized for decades as being an alarming problem in anesthetized elderly patients. Evidence confirms that approximately a quarter of all elderly having major surgery will have an identifiable fall in cognition, and $50 \%$ of these patients will suffer a permanent dysfunction.

POCD refers to disorders affecting orientation, attention, perception, consciousness, and judgment that develop after surgery. Though the precise etiology of POCD is yet to be understood, the underlying biological basis is believed to be a cholinergic failure within the central nervous system (CNS), and the cluster of symptoms is very similar to Alzheimer's form of dementia. This is thought to be the result of a malignant neuro-inflammatory response and subsequent synaptic impairment in the elderly and
Correspondence: Nalini Kotekar Department of Anaesthesiology, Critical Care and Pain Medicine, JSS Academy of Higher Education and Research Hospital, M.G. Road, Mysore, Karnataka 570004, India

Tel +91 9845112283

Email nalinikotekar@gmail.com
Clinical Interventions in Aging 2018:1 3 2267-2273

2267

Dovepress if in 0

http://dx.doi.org/1 0.2147/CIA.S133896 (c) (i) (5) 2018 Kotekar et al. This work is published and licensed by Dove Medical Press limited. The full terms of this license are available at https://www.dovepress.com/terms.php (c) $\mathrm{BY}$ and incorporate the Creative Commons Attribution - Non Commercial (unported, v3.0) License (http://creativecommons.org/licenses/by-nc/3.0/). By accessing the work you hereby accept the Terms. Non-commercial uses of the work are permitted without any further permission from Dove Medical Press Limited, provided the work is properly attributed. For permission for commercial use of this work, please see paragraphs 4.2 and 5 of our Terms (https://www.dovepress.com/terms.php). 
susceptible individuals., ${ }^{2,3}$ As a result, strategies are being explored to prevent surgery- and anesthesia-induced cognitive impairment with regard to choice and depth of anesthesia, perioperative anesthetic and other medications, and surgical strategy. The extent of cognitive deterioration subsequent to surgery and anesthesia has a significant impact on patient health and is associated with prolonged hospital recovery, greater morbidity, and delays in functional recovery. ${ }^{4}$

\section{Incidence and diagnosis}

The incidence of POCD varies depending on the cognitive performance tests, time of postoperative assessment, and the limitations of specificity and sensitivity of the current cognitive tests. The incidence after cardiac surgery is reported to be $30 \%-80 \%$ few weeks after surgery and $10 \%-60 \%$ after 3-6 months. ${ }^{5,6}$ Patients over the age of 65 years who underwent non-cardiac surgery had a $26 \%$ prevalence of POCD within few weeks which decreased to $10 \% 3$ months postoperatively. ${ }^{7}$

Diagnosis of POCD is confirmed by performing baseline cognitive performance tests prior to surgery and comparing the cognitive status following surgery. In 1955, Bedford was the first to report that general anesthesia produces longterm cognitive dysfunction in the elderly. Numerous studies followed thereafter but were inconclusive because of lack of control groups and formal neuro-psychometric tests. Subsequently, The International Study of Post-operative Cognitive Dysfunction-1 undertook multicentric studies in patients undergoing noncardiac surgeries ${ }^{8}$ and concluded that elderly patients were vulnerable for short-term cognitive dysfunction after surgery and anesthesia.

\section{Risk factors of developing POCD and preventive strategies}

POCD is a complicated entity with subjective variability and numerous contributory factors. The causes and current preventive strategies are grouped into patient-, surgical-, and anesthesia-related factors. ${ }^{9}$

\section{Patient factors}

Age, educational level, mental health, and comorbidities are contributory patient factors. Elderly patients undergoing cardiac and non-cardiac surgery are at increasing risk of developing POCD with advancing age., ${ }^{4,8}$ Aging induces degenerative changes in the brain which predisposes them to POCD for longer periods of time postoperatively. ${ }^{2}$ Patients with a level of education more than high school have a lower incidence of POCD compared to those with lower educational levels. In educated population, brain is exposed to continuous challenging mental activities that could delay the manifestations of dementia by utilizing neuronal reserves and increasing the efficacy of synapses to re-route around damaged areas. A hypothetical construct termed "cognitive reserve" has been utilized to describe models of cognitive aging. Surrogates of cognitive reserve have included educational reserve, occupational attainments, and performance on tests of knowledge such as vocabulary. ${ }^{4}$ Social support and quality of medical care are interrelated to educational advancement. Creating awareness on the benefits of healthy aging, including the importance of maintaining mental agility, may go a long way in protecting the future geriatric population from POCD.

A structured approach in preoperative assessment in geriatric patients termed as "comprehensive geriatric assessment (CGA) is now in practice, and it assesses comorbidity, cardio respiratory reserve, frailty, and health in a systematic manner. CGA allows objective assessment across multiple domains, that is, medical, psycho-social, and functional and provides scope for goal-oriented interventions. Preoperative assessment often stops at the risk assessment stage; however, it should be seen as an opportunity to optimize organ-specific disease and comorbidities. Most geriatric studies have excluded aged individuals with multiple comorbidities and major depression or cognitive disorders, thereby limiting the data available on the multivariate analysis system. Even though a battery of neurodegenerative tests remains the primary method for diagnosing POCD, there is a role for novel biomarkers and neuro-imaging techniques to preemptively identify patients at risk for cognitive decline in the perioperative period. An unexplored category in this area of study are the genetic factors as there is a strong possibility that certain patients are predisposed to POCD by virtue of their genetic profile. The only gene investigated so far is the apolipoprotein E4 (apoE4), a gene which has shown greater propensity of the brain which is vulnerable to various insults such as Alzheimer's disease and traumatic brain injury. ${ }^{10,11}$

\section{Surgery- and anesthesia-related factors}

Minor surgical procedures performed on an outpatient basis with minimal postoperative hospitalization have low risk in the elderly for developing POCD. As the duration and complexity of surgery increase so do the incidence and severity of POCD. The reason for this association includes the release of endotoxin and cerebral emboli. Endotoxins stimulate the release of interleukins which are responsible 
for systemic inflammatory response. An age-related reduced immune response to endotoxin may predispose the patients to develop POCD. As patients have become older and more sick, the incidence of brain injury after cardiac surgery has remained relatively constant over the years despite improvements in surgical techniques and implementation of effective brain protection strategies. The combination of three factors that lead to cerebral consequences after cardiac surgeries are embolism, hypoperfusion, and inflammatory response. Studies in patients who undergo cardiac surgery show a relationship between low preoperative levels of an anti-endotoxin core antibody and POCD. This suggests that a reduced immune response to endotoxin may be responsible for the development of POCD. ${ }^{12}$ In orthopedic surgery, cerebral micro emboli, in particular fatty emboli, that are common during procedures such as total knee replacements reach the brain via paradoxical embolization through a patent foramen ovale or the pulmonary vasculature ${ }^{13,14}$

The concept of Enhanced Recovery After Surgery (ERAS) also known as "accelerated recovery" stemmed from the idea of "stress free anesthesia and surgery" proposed by Henrick Kehlet in early 1990s. ${ }^{15}$ Enhanced Recovery (ER) protocols or "fast track" programs are evidence-based care bundles that have a salutary effect on postoperative recovery. These protocols include preoperative, intraoperative, and postoperative components. The efficacy of ER protocols has been established in multiple randomized controlled trials and meta-analyses, including a Cochrane review. ${ }^{16,17}$ Among the main principles, restrictive intravenous fluid therapy, use of laparoscopy in combination with appropriate anesthesia, analgesia with early enteral feeding, and early postoperative mobilization have been given importance. Majority of research on ER pertains to colorectal surgery and has also been adopted in pancreatic, gastric, urological, vascular, thoracic, gynecological, hepatobiliary, cancer, and orthopedic surgeries. It has been clearly shown that ERAS program could enhance postoperative recovery, shorten length of hospital stay, and reduce morbidity by adopting a set of optimization measures recommended by evidence-based medicine. ${ }^{18,19}$ These benefits have been attributed to attenuation of perioperative neuro-endocrine stress response, maintenance of organ function, and an accelerated return of gut function. The preoperative ERAS program gives priority to optimization of chronic illnesses to achieve best possible physical status, consultant-led anesthetic assessment, preoperative counseling, and prehabilitation. Surgery involves stress, immobilization, and unpredictable lengths of stay in unfamiliar environment, all of which have an association with deterioration of cognition in the elderly. There is substantial evidence that moderate physical activity and social involvement have a positive role in improving cognitive functions in healthy as well as in cognitively impaired elderly and that an immobile, depressed, and socially isolated geriatric surgical patient is more likely to suffer cognitive impairment and become dependent postoperatively. ${ }^{20}$

ER has evolved a novel concept of "Prehabilitation," and it refers to improving the preoperative functional state of a patient by a multimodal system involving appropriate exercise program, nutritional supplementation, and education for a period of 6-8 weeks prior to major surgery. ${ }^{21}$ This proactive approach aims at improving the functional capacity by inspiratory muscle training, simple activities, and resistance training with an aim to reduce postoperative complications. Preoperative oral nutrition supplements are provided for a period of 7-10 days and anemia if present is corrected. It is well understood that poor preoperative nutritional status translates to suboptimal surgical outcomes and increases postoperative morbidity in the aged. The ER program encourages the involvement of the elderly patient and time is taken to counsel him and allay his anxiety. Counseling helps the elderly to handle the anxiety, fatigue, and pain so as to promote early recovery and discharge. The patients are educated on the detrimental effects of smoking and alcoholism on postoperative recovery, and active measures are taken to correct both.

\section{The immediate preoperative period}

Current fasting guidelines have cut the hours of fasting because not only are long fasting periods a cause for anxiety and discomfort to the patients, it also increases the stress response and worsens catabolism. Six hours for solids and 2 hours for clear liquids work very well for ER and such patients remain euvolemic and well hydrated, with less requirement for intravenous fluids. Oral carbohydrate preloading is done with a clear carbohydrate beverage $(100 \mathrm{mg}$ of carbohydrate) on the night before the surgery and 3 hours prior to surgery. Carbohydrate preloading attenuates the neuroendocrine stress response, catabolism, and insulin resistance and improves well-being. Thromboprophylaxis is recommended for all patients starting with a single dose of enoxaparin $20 \mathrm{mg}$ the night before surgery and continued for the duration of hospitalization. Use of intermittent pneumatic compression device and compression stockings followed by early mobilization is recommended. The risk of bleeding is evaluated and weighed against the risk of venous 
thromboembolism. Antibiotic prophylaxis is given within 60 minutes of incision to the skin.

\section{Intraoperative and postoperative period}

Routine preanesthetic medication with sedatives and anxiolytics are no longer mandated. Anesthetic technique is that of balanced anesthesia with the use of short-acting opioids (remifentanyl/fentanyl).

An important preventive strategy against POCD is the monitoring of anesthetic depth intraoperatively along with that of cerebral oxygenation. Modulation of anesthetic depth could potentially minimize the anesthetic insult.

Apart from anesthetic depth, the molecular size of anesthetics is now understood to be of significance. ${ }^{22}$ Smaller size anesthetic agents such as isoflurane and desflurane may cause greater amyloid $\beta$ oligomerization. Large-size anesthetics, such as propofol, when co-administered with small-size anesthetic agents such as halothane, isoflurane, and desflurane also resulted in oligomerization, thereby adding to POCD pathology. Sevoflurane or even desflurane has been hypothesized as safe alternatives to isoflurane. ${ }^{23}$ Sevoflurane, desflurane, intravenous thiopental, and propofol infusion are preferred agents in preventing POCD. Nitrous oxide is preferably avoided especially for long duration. Laparoscopic surgeries are preferred so as to avoid bowel distension and postoperative nausea and vomiting. Lung protective ventilation with low tidal volumes $(5-7 \mathrm{~mL} / \mathrm{kg})$ is recommended. ${ }^{24}$ Epidural analgesia supplementation for open procedures is highly recommended. High inspired oxygen, when administered, improves blood flow at anastomotic site and reduces the risk of delayed wound healing. Supplemental oxygen is shown to reduce postoperative nausea and vomiting and is not associated with clinically significant side effects. Insertion of drains and nasogastric tubes are avoided to help early mobilization of patients.

Fluid therapy is goal-directed, and fluid overloading should be avoided as it is known to be associated with poor healing of intestinal anastomosis, prolonged ileus, and pulmonary and peripheral edema. Blood loss and maintenance requirements should guide the judicious fluid therapy. Hypotension associated with central neuraxial blockade and general anesthesia should be managed with vasoconstrictors rather than fluids. ${ }^{25}$ Real-time hemodynamic monitoring with esophageal Doppler is of value in the elderly patients. Postoperative early initiation of oral fluids 2 hours after surgery and tapering off intravenous fluids by the second postoperative day reduce anastomotic dehiscence, infection, and length of hospital stay. Normal saline is best avoided as it is associated with sodium overload, metabolic acidosis, and hyperchloremia leading to unfavorable outcomes. A balanced salt solution is the ideal choice of fluid.

Hypothermia in the perioperative period leads to increased metabolic demand, altered drug metabolism, impaired immunity, and coagulation. Normothermia should be maintained using temperature monitoring and use of warming devices.

Postoperative period should include multimodal analgesic approach with cautious use of opioids that tend to cause nausea, vomiting, drowsiness, and respiratory depression. All of these can cause delay in discharge. Use of epidural anesthesia reduces stress response associated with surgery and provides excellent pain management. Epidural catheters should be removed on second postoperative day to allow ease of mobilization. Paracetamol and nonsteroidal anti-inflammatory drugs should be used in preference to opioids. Local wound infiltration with local anesthetics provides excellent pain relief and lowers the rates of urinary retention. Transverse abdominal plane blocks are popular as alternate options to wound infiltration or epidural analgesia in abdominal surgeries.

Elderly patients should be nursed in a quiet postoperative environment, preferably in the presence of family members as this has proven benefits against POCD.

Postoperative hyperglycemia is known to increase the length of hospital stay and mortality rates. Regular blood glucose monitoring should aim at maintaining blood sugar levels between 180 and $200 \mathrm{mg} / \mathrm{dL}$.

Early postoperative mobilization, possibly with the assistance of a physiotherapist, reduces the risk of thromboembolic and pulmonary complications. Avoidance of nasogastric tubes, abdominal drains, and opioids assist in early mobilization and hence discharge from hospital, thereby promoting patient autonomy. Elderly patients often live alone; hence, they have to be fit enough to manage their home situations by themselves.

Surgery involves stress, immobilization, and unfamiliar surroundings, all of which have been implicated with deterioration of higher mental functions.

\section{Atrial fibrillation (AF) and cognitive decline}

Aging causes a depletion in the atrial pacemaker cells, thereby making AF the default rhythm for the elderly. The rapid ventricular rate in AF results in inadequate and variable diastolic filling and a reduced cardiac output that is poorly 
tolerated in elderly patients. AF is a common disease in the geriatric population, conferring considerable morbidity and mortality related to cardiovascular effects and thromboembolic risks. There is a growing evidence which links AF as a risk factor for POCD. ${ }^{26}$ Current treatment modalities include anticoagulation, anti-arrhythmic medications, radiofrequency ablation, and evolving surgical techniques.

Ablation for AF has been found to be a highly effective strategy; however, it has been associated with a 13\%-20\% prevalence of POCD in patients at long-term follow-up. These results were seen in a patient population with predominant $\mathrm{CHADS}_{2}$ (congestive heart failure, age $>75$ years, diabetes mellitus, previous stroke/transient ischemic attack) scores of $0-1$, representing a major segment of the elderly undergoing ablation for AF. Prolonged placement of left atrial catheters and atrial endocardial damage caused by ablation may trigger thrombus formation despite anticoagulation medication. MRI studies have depicted new cerebral lesions after irrigated AF ablation in $7 \%-14 \%$ of previously asymptomatic patients. ${ }^{27,28}$ However, the multifactorial etiology of POCD that is, the surgical procedure, anesthetic exposure, and patient susceptibility should also be taken into consideration. ${ }^{29}$ Further studies are required to assess the long-term implications of these subtle changes.

\section{The future}

Research is in progress to investigate the biomarkers of neural injury in POCD. Biomarkers such as inflammatory cytokines, amyloid- $\beta$, and tau deposition in the cerebrospinal fluid have gained attention. S100 $\beta$ and neuron-specific enolase are specific biomarkers which need to be researched. Pharmacological strategies developed for symptomatic treatment of Alzheimer's disease could be of importance in treating POCD. Cholinergic neurons are strongly impaired in Alzheimer's disease, and four cholinesterase inhibitors (tacrine, rivastigmine, galantamine, and donepezil) have been licensed to slow down the breakdown of acetylcholine in the synaptic clefts, thereby improving cognitive processes. ${ }^{30}$ These agents are effective in reducing cognitive impairment and improving activities of daily living; however, their effectiveness in POCD is yet to be proved. ${ }^{31}$ Memantine an N- methyl-D aspartic acid antagonist plays a neuroprotective role against neuronal degeneration of amyloid beta $(A \beta)$. It inhibits excessive glutamate activation and excitotoxicity caused by high concentration of glutamate, which causes cell death following injury and thereby improves cognition. ${ }^{32}$ Long-term positive outcomes for both memantine and acetylcholineesterase inhibitors have been found in terms of delayed time to death. ${ }^{33,34}$ Huperzine A, a more potent cholinesterase inhibitor, has stronger penetrative ability across the bloodbrain barrier, increased oral bioavailability, and longer duration of action. It also reduces glutamate-induced toxicity and is a promising drug in this series. ${ }^{35}$ Major research suggests that these drugs are effective only in a small subset of aged patients and have debilitating side effects. Hence, preventive strategies in POCD management are gaining momentum.

Oxidative damage to the CNS accumulates over years. Intervention for oxidative stress could be explored as a primary preventive strategy for POCD as we now understand that oxidative stress is necessarily part of the post-surgical stress response. It has been hypothesized that surgery does contribute to oxidative processes in the CNS. ${ }^{36}$ Another area of interest is the modulation of microglia in synaptic protection after surgical insult. Microglia-induced neuro-inflammation has been demonstrated in Alzheimer's disease and strategies targeting microglia offer an interesting area for further study. ${ }^{37}$ Another agent BDNF has a role in increasing synaptic plasticity and thereby synaptic function. Reviews have suggested that brain-derived neurotrophic factor improved memory function, reversed age-related changes in brain and prevented cell death. Human trials are yet to begin. ${ }^{38}$

\section{Conclusion}

The best treatment for POCD is prevention, with early recognition and management of potential perioperative risk factors. Preventive strategies should involve close collaboration between surgeons, anesthesiologists, and geriatricians in order to reduce the total hospital stay by choosing the optimum surgical technique of short duration as a means to decrease inflammatory response. Advanced age has been considered as an independent risk factor. The other risk factors for developing POCD include larger and more invasive operations, duration and depth of anesthesia, hypotension, and cerebral anoxia. Intraoperative anesthetic monitoring of the depth of anesthesia can benefit the elderly by reducing the chances of developing POCD. The ERAS program could enhance postoperative recovery, shorten the length of hospital stay, and reduce morbidity by adopting series of optimization measures recommended by evidence-based medicine during the perioperative period. We are edging closer to understand acute cognitive dysfunction in the elderly following surgery, and we hope that preventive and supportive strategies evolve in the near future. Despite recent developments, significant therapeutic effects in humans are lacking ${ }^{39}$ and current findings are preliminary to decide appropriate surgical protocol in the elderly. 


\section{Disclosure}

The authors report no conflicts of interest in this work.

\section{References}

1. Dodds C, Kumar CM, Servin F. Chapter 13: Non-theatre anaesthesia and the elderly. Cognitive Dysfunction and Sleep Disorders. Anaesthesia for Elderly Patients. Oxford Anaesthesia Library: Oxford, UK: Oxford University Press; 2017:113-123.

2. Dodds C, Allison J. Postoperative cognitive deficit in the elderly surgical patient. Br J Anaesth. 1998;81(3):449-462.

3. Kotekar N, Kuruvilla CS, Murthy V. Post-operative cognitive dysfunction in the elderly: a prospective clinical study. Indian J Anaesth. 2014;58(3):263.

4. Newman MF, Kirchner JL, Phillips-Bute B, et al. Longitudinal assessment of neurocognitive function after coronary-artery bypass surgery. N Engl J Med. 2001;344(6):395-402.

5. van Harten AE, Scheeren TW, Absalom AR. A review of postoperative cognitive dysfunction and neuroinflammation associated with cardiac surgery and anaesthesia. Anaesthesia. 2012;67(3):280-293.

6. Wu CL, Hsu W, Richman JM, Raja SN. Postoperative cognitive function as an outcome of regional anesthesia and analgesia. Reg Anesth Pain Med. 2004;29(3):257-268.

7. Brown EN, Pardon PL. The aging brain and anaesthesia. Curr Opin Anaesthesiol. 2013;26:414-419.

8. Moller JT, Cluitmans P, Rasmussen LS, et al. Long-term postoperative cognitive dysfunction in the elderly: ISPOCD1 study. Lancet. 1998;351(9106):857-861.

9. Culley DJ, Monk TG, Cosby G. Post-operative Central Nervous System Dysfunction, Geriatric Anaesthesiology. 2nd ed. Springer, UK. Chapter 9:123-136.

10. Sundström A, Marklund P, Nilsson LG, et al. APOE influences on neuropsychological function after mild head injury: within-person comparisons. Neurology. 2004;62(11):1963-1966.

11. Abildstrom H, Christiansen M, Siersma VD, Rasmussen LS, ISPOCD2 Investigators. Apolipoprotein E genotype and cognitive dysfunction after noncardiac surgery. Anesthesiology. 2004;101(4):855-861.

12. Hindman BJ. Emboli, inflammation, and CNS impairment: an overview. Heart Surg Forum. 2002;5(3):249-253.

13. Sulek CA, Davies LK, Enneking KF, Gearen PA, Lobato EB. Cerebral microembolism diagnosed by transcranial doppler during total knee arthroplasty. Anesthesiology. 1999;91(3):672.

14. Ogino Y, Tatsuoka Y, Matsuoka R, et al. Cerebral infarction after deflation of a pneumatic tourniquet during total knee replacement. Anesthesiology. 1999;90(1):297-298.

15. Kehlet H. The surgical stress response: should it be prevented? Can J Surg. 1991;34(6):565-567.

16. Lv L, Shao YF, Zhou YB. The enhanced recovery after surgery (ERAS) pathway for patients undergoing colorectal surgery: an update of meta-analysis of randomized controlled trials. Int $J$ Colorectal Dis. 2012;27(12):1549-1554.

17. Spanjersberg WR, Reurings J, Keus F, van Laarhoven CJ. Fast track surgery versus conventional recovery strategies for colorectal surgery. Cochrane Database Syst Rev. 2011;2(2):CD007635.

18. Sethi AK, Kochhar A, Ahmad Z. Enhanced Recovery After Surgery. In: Sehgal R, Trikha A, editors. Yearbook of Anesthesiology-6. New Delhi, India: Jaypee Brothers Medical Publishers (P) Ltd; 2017: $152-163$.

19. Khan S, Gatt M, Horgan A, et al. ASGBI: Issues in professional practise guidelines for implementation of enhanced recovery protocols. ASGBI, London; 2009. Available from: http://www.asgbi.org.uk/en/ publications/issues_in_professional_practice.cfm. Accessed October $31,2018$.
20. Heyn P, Abreu BC, Ottenbacher KJ. The effects of exercise training on elderly persons with cognitive impairment and dementia: a metaanalysis. Arch Phys Med Rehabil. 2004;85(10):1694-1704.

21. Moran J, Guinan E, Mccormick P, et al. The ability of prehabilitation to influence postoperative outcome after intra-abdominal operation: A systematic review and meta-analysis. Surgery. 2016;160(5):1189-1201.

22. Mandal PK, Simplaceanu V, Fodale V. Intravenous anesthetic diazepam does not induce amyloid-beta peptide oligomerization but diazepam co-administered with halothane oligomerizes amyloid-beta peptide: an NMR study. J Alzheimers Dis. 2010;20(1):127-134.

23. Mandal PK, Bhavesh NS, Chauhan VS, Fodale V. NMR investigations of amyloid- $\beta$ peptide interactions with propofol at clinically relevant concentrations with and without aqueous halothane solution. J Alzheimers Dis. 2010;21(4):1303-1309.

24. Futier E, Constantin J-M, Paugam-Burtz C, et al. A trial of intraoperative low-tidal-volume ventilation in abdominal surgery. Survey of Anesthesiology. 2014;58(4):169-171.

25. Wuethrich PY, Burkhard FC, Thalmann GN, Stueber F, Studer UE. Restrictive deferred hydration combined with preemptive norepinephrine infusion during radical cystectomy reduces postoperative complications and hospitalization time: a randomized clinical trial. Anesthesiology. 2014;120(2):365-377.

26. Hui DS, Morley JE, Mikolajczak PC, Lee R. Atrial fibrillation: A major risk factor for cognitive decline. Am Heart J. 2015;169(4):448-456.

27. Gaita F, Caponi D, Pianelli M, et al. Radiofrequency catheter ablation of atrial fibrillation: a cause of silent thromboembolism? Magnetic resonance imaging assessment of cerebral thromboembolism in patients undergoing ablation of atrial fibrillation. Circulation. 2010;122(17): 1667-1673.

28. Schrickel JW, Lickfett L, Lewalter T, et al. Incidence and predictors of silent cerebral embolism during pulmonary vein catheter ablation for atrial fibrillation. Europace. 2010;12(1):52-57.

29. Medi C, Evered L, Silbert B, et al. Subtle post-procedural cognitive dysfunction after atrial fibrillation ablation. J Am Coll Cardiol. 2013; 62(6):531-539.

30. Pepeu G, Giovannini MG. Cholinesterase inhibitors and memory. Chem Biol Interact. 2010;187(1-3):403-408.

31. Birks J. Cholinesterase inhibitors for Alzheimer's disease. Cochrane Database Syst Rev. 2006;(1):CD005593.

32. Peskind ER, Potkin SG, Pomara N, et al. Memantine treatment in mild to moderate Alzheimer disease: a 24-week randomized, controlled trial. Am J Geriatr Psychiatry. 2006;14(8):704-715.

33. Mcshane R, Areosa Sastre A, Minakaran N, Cochrane dementia and cognitive improvement group. Memantine for dementia. Cochrane Database of Systematic Reviews. 2006;6(4):CD003154.

34. Zhu CW, Livote EE, Scarmeas N, et al. Long-term associations between cholinesterase inhibitors and memantine use and health outcomes among patients with Alzheimer's disease. Alzheimers Dement. 2013; 9(6):733-740

35. Ha GT, Wong RK, Zhang Y. Huperzine a as potential treatment of Alzheimer's disease: an assessment on chemistry, pharmacology, and clinical studies. Chem Biodivers. 2011;8(7):1189-1204.

36. Schrag M, Mueller C, Zabel M, et al. Oxidative stress in blood in Alzheimer's disease and mild cognitive impairment: a meta-analysis. Neurobiol Dis. 2013;59:100-110.

37. Streit WJ, Mrak RE, Griffin WS, Microglia GW. Microglia and neuroinflammation: a pathological perspective. J Neuroinflammation. 2004;1(1):14.

38. Lu B, Bdnf LB. BDNF and activity-dependent synaptic modulation. Learn Mem. 2003;10(2):86-98.

39. Kapila AK, Watts HR, Wang T, Ma D, Wong T MD. The impact of surgery and anesthesia on post-operative cognitive decline and Alzheimer's disease development: biomarkers and preventive strategies. J Alzheimers Dis. 2014;41(1):1-13. 
Clinical Interventions in Aging

\section{Publish your work in this journal}

Clinical Interventions in Aging is an international, peer-reviewed journal focusing on evidence-based reports on the value or lack thereof of treatments intended to prevent or delay the onset of maladaptive correlates of aging in human beings. This journal is indexed on PubMed Central, MedLine,

CAS, Scopus and the Elsevier Bibliographic databases. The manuscript management system is completely online and includes a very quick and fair peer-review system, which is all easy to use. Visit http://www.dovepress. $\mathrm{com} /$ testimonials.php to read real quotes from published authors.

Submit your manuscript here: http://www.dovepress.com/clinical-interventions-in-aging-journal 